\title{
perifèria
}

Número 7, Diciembre 2007

www. periferia.name

\section{Las muchas naturalezas en los Andes}

Ricardo Cavalcanti-Schiel - Laboratoire d'Anthropologie Sociale - EHESS, Paris ${ }^{1}$

\section{Resumen}

Si en el Occidente moderno la noción de naturaleza se conforma en contraposición lógica a la noción de cultura, las disposiciones semánticas de una y otra categoría conforman, de su parte, una específica ecología del pensamiento. Lo que planteamos en el presente artículo es que esta conformación puede no ser aplicable en otros sistemas de pensamiento. Para esto, tomamos como caso de confrontación la cosmología andina y acercamos a sus referentes una interpretación etnológica de inspiración amazonista que no le suele ser usual.

\begin{abstract}
In modern Western thought the idea of nature is constructed in logical opposition to the idea of culture, and with their semantic contents they constitute a specific ecology of mind. I argue here that this arrangement can not necessarily be applicable to others systems of thought. For this purpose I take the case of Andean cosmology and I submit it to an analytical treatment inspired by the "multiculturalistic" thesis from Amazonian ethnology.
\end{abstract}

\section{I ntroducción}

Que el par conceptual naturaleza/cultura proporcione la distinción más elemental por la que las ciencias sociales formulan, delimitan y comprenden sus objetos parece ya una evidente trivialidad. Desde los albores decimonónicos de la sociología y de la antropología, enunciar el ámbito de la cultura conlleva considerar, explícita o implícitamente, donde se detuvo la "naturaleza", o entonces de qué otro modo, es decir, por medio de qué especificidades otras, sus preceptos seguirían presuntamente funcionando en las relaciones humanas, de manera que el social, sitio y terruño de la cultura, puede incluso hacer parte de la naturaleza, pero como una naturaleza aparte (casi siempre definida por un cierto nivel de "complejidad"; una complejidad que, finalmente -es decir, para los fines de la especulación sociológica-, la vuelve autonómica, o sui generis). Desde luego, el entramado conceptual en la base no sólo de las ciencias sociales sino también de otros muchos

\footnotetext{
${ }^{1}$ Enviar correspondencia a: Ricardo Cavalcanti-Schiel, riccaval@yahoo.com.br
} 


\section{perifèria}

Número 7, Diciembre 2007

\section{www.periferia.name}

ámbitos científicos, no es una excentricidad de la razón objetiva, sino que un retoño del sistema de pensamiento que sostiene la ontología del Occidente moderno. Con la licencia de la inspiración en Roy Wagner (1975: 140-151) podríamos decir que a la invención - o a cada reinvención- de la cultura corresponde la invención - o una nueva reinvención- de la naturaleza (y viceversa); de modo que lo que se consagra en la ontología del Occidente moderno es que la idea de naturaleza se concibe forzosamente en contraposición lógica a la idea de cultura.

Ahora bien, si a esta última se adscribe, en términos más genéricos, toda forma de agencia humana, las expresiones históricas de la relación simbólica naturaleza/cultura pueden ser trazadas desde mucho antes de la modernidad. Al hacerlo desde la Grecia preclásica Pierre Hadot (2004) nos sugiere que aquella agencia humana ejercida sobre (o frente a) la naturaleza fue recurrentemente formulada según el tropo figurativo - o la "metáfora", como prefiere el autor- del "desvelamiento", literalmente, del sacársele el velo; operación que se realizaría acorde unos cuantos regímenes simbólicos más específicos -o actitudes. Lo que para Hadot caracterizaría por ejemplo la "actitud prometeica" sería la utilización "de procedimientos técnicos para arrancar de la Naturaleza sus 'secretos' con el fin de dominarla y explotarla" (:115):

En la Antigüedad la actitud prometeica se presentaba bajo tres formas: la mecánica, la magia y los bosquejos del método experimental, tres prácticas que tienen en común el hecho de buscar alcanzar efectos extraños a lo que se considera como el curso normal de la naturaleza, efectos cuyas causas escapan a aquellos que no operan según tales técnicas. Al final de la Edad Media y comienzos de los tiempos modernos estas tres prácticas se acercan y se transforman profundamente para producir el nacimiento de la ciencia experimental. La divisa del mundo moderno será entonces: 'saber es poder'; pero también: 'poder, o sea, fabricar gracias a la experimentación, es saber'. (Hadot, 2004: 115, traducción nuestra). 


\section{perifèria}

Número 7, Diciembre 2007

\section{www.periferia.name}

A todas luces, la naturaleza -y con ella, la cultura- no es una invención reciente 2 . Sin embargo, lo que quisiéramos subrayar en este juego de relaciones categoriales es precisamente lo lógico, por oposición a lo fenomenológico, es decir, no se trata, en este momento interpretativo, de especificar empíricamente un ámbito exhaustivo de la naturaleza, ni de la cultura, sino que aprehender de qué manera la forma de suponer a uno, a un tiempo excluye y especifica al otro. De este modo, las fronteras de validación de los dos conceptos no están fijadas por una fenomenología inmanente. Lo que es cultural en un momento puede ser concebido como natural en otro (y viceversa), y para esto basta accionar conceptos bien conocidos de la ciencia moderna, como innatismo (en la psicología) o adaptación (en la arqueología) -o, para el sentido inverso, el de antropogénesis o etnoecología.

Tomemos como ejemplo breve las justificativas que nos ofrece el autor de un competente ensayo sociológico sobre el cante flamenco. Al considerar el lugar epistemológico de esta sociología frente a la apreciación artística y a una consecuente "flamencología" tradicional producida a partir del influjo de la afición (el "conocimiento sensual”), Gerhard Steingress sostiene que:

El arte es el que representa la expresión sensual más amplia de la práctica humana, el que sirve de reflejo espiritual y sensual de la experiencia cotidiana del hombre, en tanto que la ciencia sólo puede explicar ciertos aspectos de la realidad por medio de la racionalidad. Esta cualidad de la ciencia sirve para desarrollar una comprensión racional más profunda sobre el arte pero no lo puede sustituir como expresión total de la experiencia del hombre, ya que el arte, según escribió Freud, es uno de los proyectos auxiliadores que sirve al hombre para soportar y superar la tragedia de la vida (...). El arte es una de las posibles reacciones humanas ante la desgracia social [etcétera, etcétera]" (Steingress, 1993: 17-18, cursivas nuestras).

\footnotetext{
2 En contra el tropo figurativo (o, si se prefiere, el "mito") de una naturaleza intocada, cierta tendencia contemporánea de la ecología cultural a maximizar la agencia antropogénica hacia la asunción fenoménica de una naturaleza irremediablemente ("naturalmente") intervenida, borrando sus límites fenomenológicos ( $y$, por ende, la frontera de su distinción a la cultura), puede no ser mucho más que una redisposición cartográfica de la incidencia empírica de las dos categorías en cuestión para re-velar (poner otra vez el velo) como "definitivamente perdida" una naturaleza global que había sido hasta entonces objeto de una praxis de desvelamiento y conquista. Si a la conquista definitiva equivaldría la pérdida, la idea de naturaleza persiste lógicamente bajo el signo ideal de la pura exterioridad.
} 


\section{perifèria}

Número 7, Diciembre 2007

www. periferia.name

Al suponer una "naturaleza humana" a partir de un presunto universalismo freudiano, el autor arroja, en términos lógicos, el arte hacia el ámbito de una determinación "natural", mientras que a la sociología le tocaría el ámbito racionalizante, artificioso, estricto, de la "cultura". La "expresión sensual" está explicada (o determinada) por el innatismo, y el arte pasa a estar comprendido en el polo más "natural" de la cultura, mientras la racionalidad científica expresaría lo que podría ser considerado el polo más reflexivo de esta misma cultura -o del Espíritu humano. Es como si la cultura, reconocida por los kantianos y románticos alemanes como "segunda naturaleza", pudiera aún estar jerarquizada en una "tercera" (o una "segunda-bis") naturaleza y estuviéramos entonces frente a un esquema conceptual semejante a una muñeca rusa. De este modo, entre "naturaleza" y "cultura" las distinciones lógicas suelen ser más que todo posicionales. Una categoría depende lógicamente de la otra, y no de una frontera empírica tajante. Lo que las distingue es precisamente lo mismo que las une: una lógica categorial de relación.

Si nos desplazamos del ámbito sintáctico hacia lo semántico, sin que descuidemos de la lógica relacional estructurante dispuesta por el primero, y buscamos lo que podría expresar un contenido mínimo para la concepción occidental moderna de "naturaleza", legatario de aquella "actitud prometeica" de que nos habla Hadot, seguramente reconoceríamos como tal todo aquello que, presente en los objetos y seres, precede a una acción investida de subjetividad, la acción que reconocemos como "humana" por antonomasia, expresión del "gesto" cultural. Donde hay sujeto no hay naturaleza; hay cultura. La antropogénesis del paisaje, por ejemplo "proposición tan recurrente en la ecología cultural contemporánea", que resta verosimilitud empírica al "mito" de la naturaleza intocada, reconforma esta naturaleza, pero no le otorga la condición de sujeto. En la cultura se encierran las muchas posibilidades de expresión de la subjetividad y, de este modo, los muchos lenguajes que organizan esta subjetividad, las muchas cosmologías, serían singularidades "culturales", mientras que la naturaleza persiste, aunque multiforme, como la exterioridad hecha de una sola materia (física), y que aquella subjetividad exclusiva "y ahora, en la modernidad, excluyente" puede "desvelar". No por 


\section{perifèria}

Número 7, Diciembre 2007

www. periferia. name

casualidad, este mapa mental, esta cartografía categorial, casi siempre nos sirve para suponer que nuestra fenoménica naturaleza vale para todas las culturas.

En suma, es por medio de esta específica "ecología del pensamiento" que los ámbitos de la naturaleza y de la cultura son para nosotros verificables y delimitables. Pero en otras sociedades esto puede simplemente no funcionar.

\section{Cosmología y naturaleza en los Andes}

Los resultados de nuestras investigaciones etnográficas en un grupo quechua de los Andes meridionales bolivianos (Cavalcanti-Schiel, 2005) subrayan como particularmente significativo un fenómeno ya bien conocido de los antropólogos en los Andes de una manera general, pero que al parecer no mereció todavía la atención de un análisis más exhaustivo de algunas de sus implicaciones cosmológicas, al menos en la perspectiva por la cual fenómenos similares (en términos de relaciones lógicas) han sido analizados en la vecina Amazonía. Planteamos que los análisis lanzados desde la etnología amazonista a propósito de la economía simbólica amerindia de la relación naturaleza/cultura pueden ser dilucidadores también para la ontología de los pueblos andinos.

Para éstos, el orden propicio y bien reglado “o, como vamos a definirlo enseguida, "reproductivo"“ de la existencia depende de una continua pactación entre los elementos del cosmos, para que, en un sentido positivo (o más bien "deseado"), las fuerzas o potencias de unos no sólo no desdeñen y no dañen a los otros, sino que puedan serles mutuamente provechosos. Esto no excluye lo que se podría reconocer también como el sentido negativo, puesto que la incidencia del daño, o sea, de una potencia "predadora", también es una posibilidad inminente, aunque no necesariamente deseada. La idea (o el ideal) de una justa medida en el ethos andino parece subordinada a una permanente ponderación, implícitamente dialógica, de lo "deseado", para los múltiples sujetos del cosmos, es decir, para todos los que puedan dispensar potencia. En términos generales, la centralidad del esfuerzo, el dispendio de potencia por estos elementos "esfuerzo que en quechua 


\section{perifèria}

Número 7, Diciembre 2007

\section{www.periferia.name}

se usa designar por la voz qallpa ${ }^{3 “, ~ e s t a ́ ~ e n ~ l a ~ b a s e ~ d e ~ t o d a ~ a c c i o ́ n ~ c o n s t r u c t i v a ~ y ~}$ productiva, como también de todo daño y enfermedad.

El intercambio de esfuerzo es asimismo la base de toda sociabilidad "o más bien "socialidad", para hablar como Marilyn Strathern (1996) ${ }^{4}$. En el ámbito humano este intercambio está enmarcado por ritos de trabajo colectivo, y se puede decir que la dirección y amplitud de los circuitos de intercambio humano de trabajo es lo que define lo que está adentro (o lo que potencialmente se incluye) y lo que esta afuera (o lo que potencialmente se excluye) de cada unidad social local. En los términos de esta permanente y contextual relación interioridad/exterioridad, el intercambio de potencia no necesariamente constructiva, como el trabajo, sino que destructiva, como la agresión física en las batallas rituales, define igualmente lo complementario que uno tiene que tomarse en cuenta para poder definirse a si mismo como unidad. Pero el esfuerzo, fuerza, potencia o energía "que es aproximadamente el campo semántico de la categoría qallpa", no es solamente un atributo humano. Todos los elementos del cosmos lo tienen: los seres de este mundo presente (kay pacha), establecido por su constitución (o "conformación") "solar", luminosa y, por consiguiente, visible; como también los seres del mundo otro paradigmático, el mundo de abajo (ukhu pacha), oscuro, para allá de la frontera de lo visible, morada de los ancestros, de los muertos, sitio y refugio de una otra era cosmológica: el pasado 5 .

\footnotetext{
3 Todas nuestras referencias terminológicas nativas serán hechas en el quechua meridional (o cuzcocollao) en razón de nuestra experiencia etnográfica específica. En este sentido, no nos preocupamos con eventuales correlaciones terminológicas con el aymara, por ejemplo. Sin embargo, muchos de los conceptos y fenómenos aquí mencionados no son derivados de nuestra especificidad etnográfica, pero conforman (o antes se expresan dentro de) una misma lógica de pensamiento.

${ }^{4}$ Al rechazar la utilidad analítica del concepto de "sociedad", reconociéndolo como una suerte de entidad hipostasiada, Marilyn Strathern (1996) sugiere el concepto de "socialidad" como la "matriz relacional" ( sin forma y sin límites) de la convivencia, mientras la "sociabilidad" (Strathern, 1988) sería la conformación moral que incide como una especificación ideal (o incluso prescriptiva) de esa convivencia.

${ }^{5}$ Los ancestros referidos son tanto las generaciones más cercanas de los linajes familiares como los ancestros míticos, o, sencillamente, los más distantes, los que incluso ya se perdieron de cualquier referencia de hereditariedad específica (y por esto pueden ser compartidos como ancestros comunes o comunales de un grupo o sea, como wak'as), y que ahora se alojan en un mundo otro, que no ocupa más el espacio de la contemporaneidad. Se podrá decir que este espacio es el pasado, o que el pasado no es tan simplemente tiempo, sino que ocupa un lugar espacial, por el cual sigue presente de un modo propio, es decir, pasa a tener un estatuto de presencia en una extensión física del cosmos. En los Andes
} 


\section{perifèria}

Número 7, Diciembre 2007

\section{www.periferia.name}

En suma, esta totalidad cosmológica de agentes comprende los humanos, animales y plantas, como también las piedras, cerros, fuentes de agua y otros elementos minerales y topográficos que revelan la intersección de este mundo con el mundo otro, y asimismo las expresiones "menos visibles" o francamente no visibles de este mundo otro, ya sean manifiestas por una materialidad indirecta, ambigua, oscilante (remolinos de viento, arco iris, rayos, fuegos fatuos, espectros fantasmales) o bien por una decidida no materialidad. Una "teoría general de la agencia" para la cosmología andina no podría detenerse en la especificación y enumeración de sujetos inmanentes (hombres, espíritus, deidades, etc), como si debiera constituir un panteón exhaustivo de héroes culturales y personajes-sociales-tipo, sino que debe partir de un antecedente lógico: el esfuerzo (o dispendio de energía) como significante de la relación. Es a partir de él que se especifican los agentes, y no el revés. Los cerros, por ejemplo, no son elegidos deidades tutelares (apus) por fuerza de una fenomenología tan autoevidente cuanto insondable, sino porque están estratégicamente dispuestos dentro de una relación necesaria que ancla el sentido de presencia (es decir, sentido de lugar en la existencia cosmológica antes que meramente territorial) y "como veremos enseguida" de perpetuidad".

En un aspecto más particular, en los Andes una especial atención parece depositada sobre los elementos liminales, intersticiales, que expresarían con más contundencia lo que está en una condición de tránsito (entre mundos o entre ámbitos agentivos) y por esto son más factibles de emanar potencias interactivas. Con ellos se debe tratar por medio de prescripciones rituales específicas so pena de que las potencias emanadas se vuelvan descontroladas y peligrosas. En un aspecto más general, el ritual no es más que el lenguaje (o específicamente un "régimen textual" “Cavalcanti-Schiel, 2005) de la interacción destinada a balizar de una manera

no sólo tiempo y espacio se fusionan en una sola categoría de pensamiento, pacha, sino que fenoménicamente son a un tiempo coetáneos y coextensivos. Esto todo significa que la noción de continuidad temporal, perpetuación o persistencia implica también la suposición de un régimen necesario de disposición espacial de la existencia.

${ }^{6}$ Acerca de nuestra expresión "existencia cosmológica antes que meramente territorial", nos remitimos a la precedencia de una determinación cosmológica sobre lo territorial, lo que no quiere decir que el aspecto territorial no sea relevante para la comprensión del significado de fenómenos etnográficos como los apus, mas que, en términos lógicos, este aspecto no es determinante sino que determinado. 


\section{perifèria}

Número 7, Diciembre 2007

\section{www.periferia.name}

productiva (o mutuamente "deseada", como decimos) el intercambio de esfuerzos. $\mathrm{Si}$ de una parte las potencias brutas de los muchos sujetos del cosmos (de los hombres incluso) son peligrosas, pueden dañar, enfermar, arruinar, emponzoñar; de otra parte, una vez sometidas al acuerdo dialógico de la justa medida, realizado por medio del ritual, pasan a manifestarse como esfuerzos productivos, como factor de sociabilidad más que de una difusa "socialidad" (Strathern, 1988, 1996) ${ }^{7}$. Son las potencias del mundo otro paradigmático, el mundo subterráneo, que hacen posible que las plantas germinen, que los animales se reproduzcan, que las aguas vengan en su debido tiempo; del mismo modo que la potencia vital de la reproducción humana y la continuidad del linaje de los ayllus ${ }^{8}$ es un legado de los ancestros, que siguen su existencia en el mundo otro y de ahí siguen también emanando su potencia “o, si se prefiere, su razón ontológica?

En suma, la reproducción de la vida o, en términos más amplios, del ordenamiento del cosmos, es resultado de los intercambios (a veces más felices, a veces no) y de las pactaciones entre los muchos sujetos de este cosmos. No obstante, los acuerdos son siempre inestables y para que sigan siendo felices necesitan ser ocasional y cíclicamente renovados, porque los muchos actores de la negociación se mueven acorde sus subjetividades propias. El calendario ritual en los Andes no es otra cosa que la permanente renovación cíclica de los pactos que permiten la reproducción biológica y social, es decir, la continuidad, la perpetuación de la vida. Lo "biológico" no está determinado afuera de la "cultura", como una exterioridad inexorable;

\footnotetext{
7 "Socialidad" que, sin embargo, otorga estatuto de realidad ontológica a los muchos agentes del cosmos, dotados de subjetividades propias.

8 En quechua el término ayllu es polisémico. Su significado más primario es el de "familia"; por extensión, el de red de alianzas parentales; por extensión, el de comunidad; y por extensión, el del territorio ocupado por un grupo local. Su quid semántico nos parece ser el de una unidad reproductiva organizada siempre bajo la lógica de la complementariedad (Cavalcanti-Schiel, 2005).

${ }^{9}$ Que no se confunda esta última expresión con una suerte de ultimátum funcionalista del significado de los entes cosmológicos. Cuando hablamos de su "razón ontológica" no estamos afirmando que la razón de existencia de estos entes (los hombres incluso) esté subordinada a un imperativo fenoménico trascendente (la reproducción, por ejemplo). Más bien, estamos afirmando que su significado sólo es posible en un contexto de relación en el que el esfuerzo (o dispendio de energía o, en la voz quechua, qallpa) es, como decimos, su significante. Y, en la misma medida en que Lévi-Strauss lo definió, se trataría aquí igualmente de un significante con "valor simbólico cero" (Lévi-Strauss, 1950), o sea, sin un contenido fenoménico prescriptivo, pero que, en su lugar, demanda siempre, contextualmente, un "contenido simbólico suplementario" (idem).
} 


\section{perifèria}

Número 7, Diciembre 2007

www. periferia. name

tampoco parece distinguirse de ella. Su posibilidad comparte de la misma materia del lenguaje: la interacción necesaria entre sujetos, es decir, depositarios de potencia y agencia.

Bajo un código general, el de la reciprocidad, el ritual es el procedimiento discursivo por el cual los acuerdos ("cósmicos" y humanos) se establecen (o, antes, se renuevan). Si el esfuerzo es el término común de la sociabilidad, la reciprocidad es su gramática, y el ritual, uno de sus más expresivos discursos. Hay otros, pero nos abstenemos de tratarlos aquí, ya sea por algo de redundancia, ya sea por la relativa exigüidad de espacio para dar cuenta de las aclaraciones necesarias, o bien porque hacerlo significaría dispersarnos por demasiados ambages frente a nuestro tema central. En lo que toca a nuestros intereses expositivos por el momento, dejamos como breve conclusión la proposición de que en la concepción andina de la existencia no hay reproducción de la vida sin que los muchos agentes del cosmos se encuentren en la condición previa de compartir referentes simbólicos, es decir, lenguaje. Lo que está dado como término del común (o del universal) no es lo que nosotros llamamos "naturaleza", sino lo que nosotros llamaríamos "cultura".

\section{Conclusión: el multinaturalismo amerindio y nuestra ecología}

Al concluir nuestras observaciones, volvemos al planteamiento del primer párrafo del tópico anterior sobre los posibles acercamientos analíticos entre Andes y Amazonía para evocar la proposición de Eduardo Viveiros de Castro acerca del concepto de "multinaturalismo". Lo característico de este concepto, cuajado por el autor a partir del material etnográfico amazónico, es el reconocimiento de subjetividades propias que las cosmologías de los pueblos indígenas de esta región asignan a los muchos agentes del cosmos, es decir, el reconocimiento de estos agentes como sujetos sociales. Así, Viveiros de Castro propone utilizar la noción de multinaturalismo

(...) para señalar uno de los rasgos contrastivos del pensamiento amerindio con relación a las cosmologías 'multiculturalistas' modernas. Mientras estas se apoyan en la implicación mutua entre unicidad de la naturaleza y multiplicidad de las culturas "la primera garantizada por la universalidad objetiva de los cuerpos y de la sustancia, la segunda generada por la particularidad subjetiva de los espíritus y del significado" la concepción amerindia supondría al 


\section{perifèria}

Número 7, Diciembre 2007

www. periferia. name

contrario una unidad del espíritu y una diversidad de los cuerpos. La cultura o el sujeto serían aquí la forma del universal; la naturaleza o el objeto, la forma del particular" (Viveiros de Castro, 2002: 348-349, traducción nuestra).

Lo que sugerimos aquí es que la cosmología andina comparte los mismos fundamentos de esta economía simbólica del multinaturalismo. Al equipararse por el universal del lenguaje y por el atributo de la subjetividad, los agentes del cosmos se distinguen muy tajantemente por sus "cuerpos", es decir, por su condición de materialidad y visibilidad. $Y$ es precisamente esto lo que les atribuye especificidad ontológica en un orden necesario de relaciones. En la disposición comparativa que tomamos por referencia, es lo que les distribuye por múltiples naturalezas. Al subrayar aquella significación ontológica establecida en un orden necesario de relaciones quitamos decididamente ${ }^{10}$ de esta multiplicidad de naturalezas la simple condición de metáfora; ella es ontológicamente constitutiva.

Conclusivamente, en términos sintéticos, reforzamos como hipótesis bastante plausible que en el pensamiento amerindio sudamericano de una forma general no es la naturaleza el término de un universal, exterior y anterior a las culturas, sino que es la "cultura" (como subjetividad y lenguaje) el término de este universal, a partir del cual los seres del cosmos se dispondrían entonces por múltiples naturalezas. En esta perspectiva, entre los pueblos indígenas andinos, un concepto nuestro como el de "conservación" -y sus consecuencias semánticas a propósito de la pasividad de una exterioridad- da lugar a una lógica agentiva de la reproducción, condición sin la cual la continuidad de la existencia no es posible. Lo que, por consiguiente, las sociedades amerindias en general nos enseñarían no es que ellas sean "naturalmente" ecologistas; sino que es nuestra específica objetificación de la naturaleza que nos exige que seamos ecologistas como quizá la sola manera de barruntar la continuidad de la existencia biológica. La ecología de la naturaleza "perdonada la redundancia" nos toca a nosotros occidentales modernos. La comprensión de las ecologías del pensamiento es lo que nos permite llegar más allá

\footnotetext{
${ }^{10}$ Ya sea en el ámbito lógico o en el ámbito fenomenológico atinente a este universo de pensamiento.
} 


\section{perifèria}

Número 7, Diciembre 2007

www.periferia. name

de nosotros mismos y nuestras aparentemente "naturales" evidencias (sobre la naturaleza, por ejemplo).

\section{Bibliografía}

Cavalcanti-Schiel, Ricardo (2005). Da relutância selvagem do pensamento. Memória social nos Andes meridionais. Rio de Janeiro: Programa de Pós-Graduação em Antropologia Social- Museu Nacional- Universidade Federal do Rio de Janeiro. Tesis de doctorado.

Hadot, Pierre (2004). Le voile d'Isis. Essai sur l'histoire de l'idée de nature. Paris: Gallimard.

Lévi-Strauss, Claude (1950). "Introduction à l'oeuvre de Marcel Mauss" en Marcel Mauss. Sociologie et anthropologie. Paris: Presses Universitaires de France, IX-LII, 1980.

Steingress, Gerhard (1993). Sociología del Cante Flamenco. Sevilla: Signatura Ediciones/ Junta de Andalucía- Consejería de Cultura, 2006.

Strathern, Marilyn (1988). The gender of the gift. Problems with Women and Problems with Society in Melanesia. Berkeley: University of California Press.

- (1996). "The concept of society is theoretically obsolete" en Tim Ingold. Key Debates in Anthropology. Londres: Routledge, pp. 60-66.

Viveiros de Castro, Eduardo B. (2002). "Perspectivismo e multinaturalismo na América Indígena" en E. Viveiros de Castro. A inconstância da alma selvagem. São Paulo: Cosac \& Naify, pp. 345-399.

Wagner, Roy (1975). The Invention of Culture. Chicago: University of Chicago Press, 1981 ("revised and expanded edition"). 